\title{
Les défis éducatifs du changement climatique : La pertinence de la dimension sociale
}

Pablo Meira et Édgar J. González Gaudiano

\section{(2) OpenEdition}

Édition électronique

URL : http://journals.openedition.org/ere/730

DOI : $10.4000 /$ ere. 730

ISSN : 2561-2271

Éditeur

Centr'ERE

\section{Édition imprimée}

Date de publication : 20 décembre 2016

ISSN : 1373-9689

\section{Référence électronique}

Pablo Meira et Édgar J. González Gaudiano, «Les défis éducatifs du changement climatique : La pertinence de la dimension sociale », Éducation relative à l'environnement [En ligne], Volume 13 - 2 | 2016, mis en ligne le 20 décembre 2016, consulté le 21 février 2020. URL : http:// journals.openedition.org/ere/730 ; DOI : 10.4000/ere.730 


\title{
Les défis éducatifs du changement climatique : La pertinence de la dimension sociale
}

\author{
Pablo Meira et Édgar J. González Gaudiano
}

\section{NOTE DE L'AUTEUR}

Cet article presente certains résultats des travaux menés dans le cadre du Projet RESCLIMA (EDU2012-33456), financé par la Dirección General de Investigación Científica y Técnica du Ministerio de Economía y Competitividad del Gobierno de España.

1 Le changement climatique fait maintenant l'objet d'une préoccupation sociale largement répandue, même si l'intérêt pour ce phénomène est relativement nouveau dans la sphère publique, étant donné qu'il n'a émergé du champ scientifique que dans les années 90. Dans ce champ, le changement climatique fait référence à une représentation scientifique qui intègre les connaissances de différentes disciplines, donnant lieu à un domaine de recherche hybride et complexe qui défie la démarche scientifique conventionnelle.

2 L'incertitude est une composante indissociable du changement climatique. Elle est associée à la difficulté d'identifier toutes les variables en cause et de concevoir comment elles sont reliées les unes aux autres, ainsi qu'au défi que pose le transfert des connaissances depuis l'échelle climatique globale vers les échelles régionales, sousrégionales ou locales afin d'identifier et d'évaluer ses impacts actuels et futurs sur l'environnement et sur l'avenir de notre humanité. Bien que l'incertitude épistémique soit inhérente à toutes les connaissances scientifiques, le cinquième rapport du Groupe d'experts intergouvernemental sur l'évolution du climat (GIEC/IPCC, 2014) affirme néanmoins que la perturbation du climat est réelle et qu'au-delà de tout doute raisonnable, elle demeure causée par l'activité humaine. 
3 Malgré l'urgence de la situation signalée depuis longtemps par la science, c'est seulement au cours de la dernière décennie que les négociations internationales pour minimiser les causes du changement climatique ont connu des avancées significatives. Par ailleurs, bien que cette question vive se soit installée progressivement dans la culture commune, le paradoxe demeure que, pendant les cinq dernières années, le changement climatique a perdu du poids dans l'ordre du jour public, comme en témoignent les agendas politiques, médiatiques, éducatifs ou sociaux (Scruggs et Benegal, 2012). Dans les pays du sud de l'Europe par exemple, la question du climat s'est écartée des préoccupations majeures de la population, perdant de sa pertinence au profit d'autres menaces de nature socio-économique ou reliées au terrorisme et aux conflits internationaux, ou encore au profit d'autres questions environnementales de plus petite envergure ou de plus grande proximité (Meira et autres, 2013 ; Commission européenne / European Commission, 2015).

4 Certes, il est possible que des initiatives récentes comme la publication de l'encyclique papale Laudato-si (Pape François, 2015) ou les attentes générées par l'Accord de Paris sur le climat (COP21, 2015) contribuent à modifier cette tendance, mais il sera difficile de centrer l'attention et l'intérêt public sur le changement climatique considérant l'importance accordée par les médias aux problèmes et menaces à court terme.

5 De plus, la version courante du concept de science, comprise comme un stock de savoir cumulatif, issu d'un processus évaluatif neutre et aseptique - non subjectif-, a dominé et domine encore largement les programmes éducatifs sur le changement climatique. De ce point de vue, la crise climatique dériverait de la méconnaissance généralisée des flux d'énergie et de matières qui circulent entre la société et la nature (Foladori, 2000), de sorte que les programmes d'éducation se transforment en processus d'alphabétisation scientifique ou, plus précisément, climatique. Ainsi, de nombreuses propositions éducatives sur le changement climatique gravitent autour d'une série de thèmes spécifiques concernant entre autres la physique de l'atmosphère, la dynamique des fluides, les principes de base de la thermodynamique, le cycle du carbone ou le rôle thermorégulateur des courants marins (Flannery, 2006).=

6 En outre, on y retrouve la prémisse selon laquelle les personnes, en acquérant des informations scientifiques, auraient tendance à modifier leur comportement, leurs attitudes et leurs valeurs et viendraient ainsi gonfler les rangs d'une population alphabétisée en matière d'environnement. Cette présomption que la source du problème réside dans un manque d'information de la population est simpliste, mécaniciste et déterministe. Des avertissements ont précédemment été formulés au sujet des limites d'une telle approche transmissive (Sterling, 2001), dont les résultats sont précaires.

7 En conséquence, la question se pose : faut-il privilégier une alphabétisation scientifique qui prétend ajuster la représentation sociale aux meilleures données scientifiques sur le climat ou une éducation environnementale centrée sur les aspects les plus significatifs pour la vie des personnes et orientée de manière à encourager des changements pertinents dans leurs modes de vie (Gilmore, 2000 ; Urbina et Martinez, 2006 ; Hempel, 2014)?

8 À l'épicentre du problème, nous retrouvons le fait que la vulgarisation scientifique sur le changement climatique imprègne la culture commune à travers des moyens et des médiateurs qui ne sont pas scientifiques ou qui n'ont pas une qualification scientifique précise (McBean et Hengevel, 2000). Ainsi, dans le transfert de la science à la société, 
une large gamme d'émetteurs (les politiciens, les journalistes, les militants sociaux, etc.) qui "transmettent", "expliquent» et "interprètent " les éléments de cette science, œuvrent en supposant que le grand public a besoin de ces connaissances pour prendre conscience du problème et transformer son mode de vie. De fait, il est attendu que l'accès à la connaissance scientifique vienne remplacer la connaissance de sens commun et fasse tomber les barrières pour entraîner les changements nécessaires, centrés sur la sphère des comportements personnels qui sont reliés à l'utilisation et la consommation de l'énergie.

Mais avant de passer à l'analyse de la complexité des programmes éducatifs sur le changement climatique, nous croyons nécessaire de procéder à certaines clarifications. Nous ne nous opposons pas à la promotion de comportements pro-environnementaux spécifiques que la population en général commence à adopter pour réduire la consommation d'énergie. Un million d'actions spécifiques réunies ont évidemment des effets significatifs sur le système climatique, positifs ou négatifs. Toutefois, nous interrogeons l'hypothèse selon laquelle c'est grâce à de tels changements que nous parviendrons à résoudre le problème et que nous nous acquitterons de notre responsabilité individuelle et collective.

Nous ne nous opposons certes pas non plus à la diffusion des connaissances scientifiques disponibles, que ce soit dans les contextes éducatifs formels ou à travers les médias et les réseaux sociaux. La science du climat est l'une des ressources les plus précieuses à notre portée pour permettre à la société de comprendre les changements atmosphériques qui se produisent et de prendre des décisions éclairées sur la façon de les atténuer et de s'adapter à leurs conséquences. Mais soutenir que l'alphabétisation scientifique assure à elle seule le changement de valeurs et de comportements dans la vie quotidienne des gens, demeure une croyance naïve (Kahan et al, 2012; Hempel, 2014).

Outre la transposition de la science vers la société, nous avons besoin d'une approche pédagogique adéquate pour introduire la science du climat dans la culture commune; il s'agit de la transformer en sens commun afin de favoriser son intégration dans la représentation sociale du changement climatique. Une telle approche pédagogique doit inviter à remettre en cause les valeurs normatives qui organisent la vie sociale; elle doit permettre d'affaiblir la résistance et les obstacles cognitifs, psychosociaux et culturels empêchant les changements, et de favoriser les actions collectives organisées autour de finalités explicites. Ainsi, les actions spécifiques pourraient prendre un autre sens et contribuer à surmonter l'état actuel des choses. Il ne s'agit pas seulement de former des individus qualifiés dans des sociétés scientifiquement cultivées, mais de construire une citoyenneté critique et consciente des implications vitales du changement climatique, et proactive dans le développement de modèles sociaux et de modes de vie alternatifs à ceux qui se retrouvent actuellement au cœur du problème.

\section{La représentation du changement climatique dans la culture commune}

12 Le changement climatique possède une série de caractéristiques qui en font un sujet essentiellement complexe, tant du point de vue scientifique que dans son enracinement dans la culture commune. Sa complexité s'exprime également dans les sphères socioéconomique, politique et éthique. La nécessité de prendre des décisions urgentes est 
confrontée à un modèle de développement dominant appuyé sur des sources d'énergie fossiles, éléments essentiels de notre mode de production et de consommation. Il est impossible de concevoir des politiques et des programmes structurels pour atténuer les effets du changement climatique sans remettre en question les aspects clés du modèle économique en vigueur et le cosmos socioculturel qui s'y rattache.

Le changement climatique est probablement la première problématique environnementale globale dont on prend conscience du caractère radicalement systémique : pratiquement tous les systèmes écologiques et humains sont impliqués et sont ou seront affectés par ses conséquences à court, moyen et long terme. Les solutions, quelle que soit l'approche adoptée - mitigation et/ou adaptation -, impliquent un changement fondamental dans la manière de transformer, distribuer et consommer l'énergie afin de réduire significativement les émissions anthropiques de gaz à effet de serre et de préserver les sources et les puits naturels de carbone.

La voie du changement est déjà identifiée, mais les résistances sont nombreuses. La même inertie sociale empêche de se mettre en mouvement. Certaines résistances tirent leur origine du mode de construction des représentations sociales du changement climatique, principalement au sein des sociétés dites plus avancées. Dans cette construction, non seulement sont impliqués la science et ses médiateurs, comme nous l'avons signalé, mais aussi les processus culturels qui répondent à une épistémologie du sens commun (Moscovici et Hewstone, 1986; Wagner, Hayes et Flores, 2011). En effet, bien que le transfert de l'information scientifique vers les citoyens exerce un rôle important, d'autres processus échappant à la logique scientifique interagissent aussi ; nous les décrirons ici de manière synthétique. Certains sont liés à la nature complexe du problème, d'autres aux processus de socialisation qui construisent le sens commun et d'autres encore, à la réinterprétation de l'information au moment de son intégration dans les représentations mentales de chaque personne, menant à travers de multiples interactions sociales, à la constitution des croyances socialement partagées.

Considérer ces processus est essentiel pour envisager correctement les défis de l'éducation au changement climatique. Nous pouvons affirmer que la principale "barrière culturelle " pour le changement est la nature structurelle du problème. À partir de cette prémisse, nous identifions des obstacles culturels, cognitifs et psychosociaux qui conditionnent la représentation sociale du problème. Pour les aborder de manière systématique, nous les avons regroupés en trois grandes catégories : A) les obstacles liés à la complexité du problème ; B) les obstacles associés aux implications morales et socio-politiques; C) les obstacles reliés aux processus cognitifs et psychosociaux.

\section{La difficile appréhension sociale de la complexité scientifique}

\section{Le changement climatique : des facteurs naturels ou des facteurs humains?}

Une des difficultés pour la compréhension du changement climatique par le public réside dans le fait qu'interviennent simultanément des facteurs naturels et humains. Les travaux du GIEC intègrent les facteurs naturels dans leurs modèles explicatifs, considérant qu'ils furent cruciaux dans la production des changements climatiques relativement brusques du passé. Ce constat alimente les arguments "négationnistes " qui contestent le changement climatique et les politiques qui en découlent: s'il s'agissait d'un phénomène d'étiologie naturelle, toute politique d'atténuation 
manquerait de sens, il faudrait ainsi se résoudre à une approche adaptative (Lomborg, 2001). Or le récent rapport du GIEC (2014) témoigne de recherche scientifiques rigoureuses qui évaluent le bilan énergétique du système climatique, en considérant l'influence de multiples facteurs (l'augmentation de la concentration de gaz à effet de serre, l'albédo terrestre, le rôle des aérosols, les fluctuations du rayonnement solaire, etc.). Selon le GIEC, même en tenant compte des facteurs naturels, il est évident que la majeure partie du changement du climat détecté est attribuable à l'activité humaine.

\section{Les causes du changement climatique : un effet agrégé}

17 Comme nous l'avons signalé, la somme des actions individuelles peut avoir des effets positifs ou négatifs sur le système climatique mondial. Une action singulière et apparemment inoffensive, comme allumer un ventilateur, peut représenter un danger si elle est répétée des millions de fois. Les émissions de gaz à effet de serre de nombreuses sources ponctuelles, pour lesquelles les « doses » considérées de manière isolée ne sont pas inquiétantes, contribuent dans leur ensemble à modifier substantiellement le système climatique. Évaluer le caractère adéquat ou inadéquat, moral ou immoral d'une action spécifique à partir du point de vue individuel de la personne qui la pose est compliqué, puisque cette appréciation dépend de contextes qu'il est difficile d'appréhender dans toute leur complexité et qui déterminent l'impact d'un geste particulier.

\section{La dimension spatiotemporelle du changement climatique}

18 Le changement climatique est un problème différé dans le temps et omniprésent dans l'espace. Du point de vue temporel et tenant compte de l'évolution naturelle du climat, le rythme du changement s'est accéléré au cours des dernières décennies. Cependant, ce changement - qui a commencé avec la révolution industrielle et dont les manifestations les plus critiques sont prévues pour la fin du XXI siècle - est perçu comme étant « lent » (Niemeyer, Petts et Hobson, 2005 ; Leiserowitz, 2005). Le manque de synchronisme entre l'échelle temporelle du changement climatique et l'échelle du temps humain entrave la prise de conscience de l'ampleur du problème et retarde la conscience du besoin urgent d'agir :

a. d'une part, le décalage entre les actions impliquant l'émission de gaz à effet de serre et ses effets sur l'atmosphère - différés et cumulatifs - empêche de relier les conduites concrètes produisant des émissions à leurs conséquences environnementales ;

b. d'autre part, l'urgence d'un changement est difficile à saisir, étant donné que ni les effets négatifs du changement climatique ni les effets positifs des actions qui réduisent les émissions ne se perçoivent instantanément.

19 Cette difficulté à saisir et à évaluer la rapidité du changement climatique fait obstacle à la compréhension d'un processus qui est lent du point de vue de la perception humaine, mais trop rapide dans une optique climatique et écologique. Il est difficile de comprendre que l'impact du changement climatique et la capacité d'adaptation humaine tout comme celle des écosystèmes dépendent de la vitesse du processus. Cette variable implique de prendre de compte la vulnérabilité différenciée des communautés humaines, de même celle des espèces et des écosystèmes dont les capacités d'adaptation répondent à des changements graduels et se manifestent à long terme. 
20 À ces difficultés s'ajoute la possibilité de transformations qui répondent à un « effet de seuil »: des altérations progressives et cumulatives, presque imperceptibles, peuvent soudain provoquer des changements brusques, rompant drastiquement la stabilité précaire antérieure. Le changement climatique ne correspond pas en effet à un phénomène linéaire. En tant qu'êtres humains, nous avons tendance à surestimer les phénomènes météorologiques extrêmes, étant donné que leurs conséquences génèrent un impact émotionnel important, mais nous négligeons souvent les changements subtils, bien que significatifs, qui se produisent dans notre environnement (par exemple, l'augmentation décimale des températures moyennes ou les changements dans les rythmes phénologiques).

21 Un autre obstacle relié à la perception du temps est la difficulté à comprendre l'irréversibilité et l'inertie du changement climatique. La méconnaissance scientifique du problème par de larges segments de la population, qui sous-estiment les risques qui menacent leur existence, est utilisée comme excuse pour reporter l'adoption de changements radicaux (O'Connor et coll., 1999; Whitmarsh, 2011). Or la science du climat avise que plus les mesures pour réduire les émissions de gaz à effet de serre sont retardées, plus les conséquences du changement climatique auront tendance à être profondes et irréversibles à long terme, à la fois pour l'environnement biophysique et les communautés humaines (GIEC, 2014).

D'un point de vue spatial, l'ubiquité du changement climatique crée également des problèmes pour sa représentation sociale. Il est difficile, même pour la science, de préciser comment le changement climatique affecte présentement ou comment il affectera l'avenir de chaque région de la planète. Bien que dans le récent rapport du GIEC, l'incertitude à propos des scénarios régionaux prévisibles ait été grandement réduite, les prédictions perdent de leur fiabilité au fur et à mesure qu'est précisé le territoire sur lequel elles sont projetées, ajoutant plus d'incertitude encore à la perception sociale du problème.

23 En outre, le fait que les effets ne soient pas équivalents dans les différentes zones de la planète contraste avec l'idée habituellement transmise, associant le changement climatique au réchauffement de l'ensemble de la Terre. Ce qui est certain c'est que les températures moyennes à des points localisés de la surface terrestre peuvent refléter cette augmentation, mais peuvent aussi se situer au-dessus ou en-dessous de cette tendance. Ainsi, les prédictions globales diffusées par les médias peuvent contredire l'expérience locale (Morgan et al, 2002 ; Hitchings, 2011). Par exemple, le changement climatique peut être associé à une plus faible disponibilité globale d'eau douce, même si certaines communautés humaines souffriront d'inondations récurrentes, jusqu'alors inaccoutumées pour eux.

24 En raison de cette double perspective, temporelle et spatiale, le changement climatique peut apparaître à la population comme un problème abstrait et intemporel, comme un phénomène contrintuitif dont les causes ne sont pas faciles à cerner et dont les conséquences se projettent sur une échelle dépassant l'horizon de vie immédiat (Uzzell, 2000 ; Spence et coll., 2012).

\section{L'incertitude : son sens scientifique et social}

25 L'incertitude est consubstantielle à la connaissance scientifique. Celle dernière ne peut évoluer qu'en se soumettant à une révision constante, à la controverse et au débat par 
et au sein de la communauté d'experts qui la développe. L'incertitude inhérente à la connaissance $\mathrm{du}$ changement climatique rend particulièrement difficile la communication avec la société. Il s'agit en effet d'une construction scientifique caractérisée par son énorme complexité: le changement climatique implique de multiples systèmes et variables en relation synergique; il est abordé à partir de différentes disciplines et plusieurs lacunes importantes existent à propos d'aspects fondamentaux qui constituent sa genèse.

Si l'incertitude épistémique est inhérente au processus scientifique, sa projection sociale crée de la confusion, entrave la prise de conscience de la gravité du problème et de l'urgence d'agir. L'insistance sur les marges d'indétermination du phénomène du changement climatique décourage les approches préventives et alimente l'optimisme historique basé sur l'idée de progrès, qui soutient que l'avenir sera nécessairement meilleur (Barnes, 1996). L'économie de l'effort qui régit la vie quotidienne trouve dans l'incertitude - projetée volontairement ou involontairement par la science - une excuse pour relativiser la gravité de la menace et retarder l'action (Heras et Meira, 2014).

L'incertitude dans la construction scientifique du changement climatique est transférée à la société de différentes façons :

a. La science implique une démarche méthodologique qui inclut la validation collégiale des connaissances scientifiques. Du point de vue des citoyens et citoyennes profanes, cependant, l'écho public des controverses propres au débat scientifique peut être interprété comme une insécurité, une confusion ou une division au sein de la communauté scientifique, alimentant des doutes quant à l'existence du problème, ses causes et ses conséquences possibles, compromettant ainsi l'importance qu'elle mérite en tant que menace réelle (Zehr, 2000; Whitmarsh, 2011). L'incertitude scientifique peut être manipulée pour nourrir le « négationnisme » et l'inaction.

b. b) La tendance des médias - principales sources d'informations environnementales pour les citoyens et citoyennes - visant à donner la même couverture aux voix scientifiques qui sonnent l'alarme à propos de la menace climatique - qui sont majoritaires - qu'aux voix minoritaires de scientifiques, d'institutions et de créateurs d'opinion qui la nient, l'attribuent à des facteurs naturels ou la sous-estiment en interpelant la capacité d'adaptation des écosystèmes et des sociétés, exerce également une grande influence (Boykoff, 2011).

c. Un autre facteur qui augmente l'incertitude est la tendance à mettre l'accent sur les lacunes dans les connaissances climatiques plutôt que sur les certitudes confirmées ou identifiées comme les plus probables (Clark, Stamm et Reynolds, 1998 ; Urbina et Martinez, 2006). Il est important de considérer que plus nous en savons sur le climat et le changement climatique, plus des lacunes dans notre connaissance du phénomène sont identifiées, pour lesquelles la science doit poursuivre ses recherches.

d. Le même effet se produit avec l'accent mis sur les marges de variabilité dans les modèles de prédictions pour les scénarios climatiques de l'avenir, au détriment d'une mise en évidence de la probabilité toujours croissante que les pires scénarios envisagés se convertissent en réalité, exactement comme nous en avise le GIEC (2014). 


\section{L'expression socio-politique et morale du changement climatique}

\section{De la complexité scientifique à la complexité sociale} Premièrement, il est l'un des "produits » les plus saillants de l'universalisation du modèle de production né avec la révolution industrielle. Deuxièmement, il devient clair que ce modèle a induit une crise qui découle de son propre succès en tant que mode d'organisation de la production matérielle, de création d'un environnement relativement sécuritaire et de production de significations et de modes de vie partagés par une partie importante de l'humanité. Et, troisièmement, les solutions, quelles qu'elles soient, doivent être associées à un haut niveau de consensus international, parce que pour être efficaces elles, elles doivent être assumées et mises en œuvre à l'échelle mondiale. Ainsi, la complexité sociopolitique se superpose à la complexité scientifique. En d'autres termes, un effet synergique se produit, donnant lieu à une crise hybride où s'amalgament les sphères scientifique, sociale et politique dans une tension permanente.

D'autre part, la complexité entourant cette menace s'exprime également dans le champ moral. La responsabilité humaine envers les causes du changement climatique est inégalement répartie, à la fois globalement, comme au sein de chaque société spécifique. La plupart des émissions historiques et actuelles de gaz à effet de serre ont été et demeurent générées par les pays les plus développés. Dans le scénario d'un marché délocalisé, une grande partie des émissions du monde sous-développé est générée pour satisfaire les besoins et les désirs des habitants des pays dits les plus « développés ».

Ainsi, les causes et les conséquences du changement climatique apparaissent étroitement associées à la croissance des inégalités dans le développement humain et aux dilemmes éthiques, politiques et idéologiques impliqués. Les sociétés pauvres sont les plus vulnérables face aux perturbations climatiques. Une grande partie de leur potentiel de développement repose sur les activités extractives et sur la transformation des ressources - énergétiques, minérales, forestières, agricoles, etc. - figurant parmi les activités humaines qui perturbent le plus le climat. De plus, ces sociétés ont moins de ressources à leur disposition pour prévenir ou atténuer les conséquences du changement et sont les plus vulnérables devant les impacts indirects de la modification des écosystèmes, les dysfonctionnements économiques, l'émergence de nouvelles menaces pour la santé ou la dégradation de ressources vitales telles que le sol ou l'eau.

De l'autre côté, les sociétés les plus développées, qui totalisent un peu plus du cinquième de la population mondiale, accumulent par personne les taux les plus élevés de consommation de combustibles fossiles et d'émissions de gaz à effet de serre (Martínez Alier, 2007). En accentuant les inégalités, le changement climatique pose des dilemmes moraux difficiles à résoudre dans le contexte actuel d'une crise économique systémique et d'une scène géopolitique internationale de plus en plus complexe. Les rondes infructueuses de négociations internationales visant à convenir d'un protocole post-Kyoto comprenant des engagements spécifiques de réduction des émissions pour les pays comme l'Inde, la Chine, le Mexique et le Brésil, jusqu'à aujourd'hui exclus de cette obligation, ou visant à faire en sorte que les pays plus "développés » assument leur responsabilité principale comme générateurs du problème, mettent en évidence la difficulté à résoudre ces dilemmes. On peut espérer que la COP21 permette de sortir

Éducation relative à l'environnement, Volume 13 - 2 | 2016 
d'une telle paralysie, mais tout indique qu'au-delà des discours positifs des acteurs qui représentent les principaux intérêts en jeu, il sera difficile de mettre en œuvre les accords contraignants qui permettraient de réduire les gaz à effets de serre de façon à limiter la hausse moyenne des températures à $2^{\circ}$ centigrades, ce qui est considéré comme le moins pire des scénarios pour la fin de ce siècle.

\section{L'action dans le labyrinthe}

Le fait que nos contributions individuelles au changement climatique soient souvent imperceptibles et diffuses rend difficile la reconnaissance de notre propre apport au problème ainsi que de notre capacité à contribuer à le résoudre. Le labyrinthe éthique et social dilue la responsabilité individuelle et empêche de considérer adéquatement les différents rôles que nous jouons en relation avec le problème - consommateurs, citoyens, agriculteurs, professionnels, etc. Par exemple, beaucoup de gens ont une vision déformée du modèle énergétique actuel et sont incapables de juger de leur rôle dans celui-ci : ils ne savent pas d'où l'énergie consommée provient ni comment elle est produite. Ils ignorent largement comment et en quelle quantité elle est consommée et ils n'identifient pas les conséquences que leur comportement énergétique individuel entraîne pour l'environnement, le climat et les personnes, celles qui vivent actuellement et celles des générations à venir (Bedolla et Moreno, 2011 ; Velayos, 2008).

À ce fait, il faut ajouter les distorsions introduites par le marché, étant donné que le prix de l'énergie n'est pas toujours un indicateur fiable de la rareté relative de la ressource ni des coûts sociaux et environnementaux de leur production. Dans cette situation, il est difficile de planifier des changements à propos de quelque chose de méconnu ou de ce dont nous n'avons qu'une vision et une expérience partielle, incomplète et déformée.

\section{La multicausalité invisible}

34 Les sociétés « développées » - et aussi, de plus en plus, celles qui ne le ne sont pas, mais aspirent à l'être en les imitant - appuient leur fonctionnement sur la consommation de grandes quantités d'énergie provenant surtout de sources fossiles. Cette voracité énergétique fondamentale rend pratiquement toutes nos actions - même lorsque nous dormons - génératrices d'émissions de gaz à effet de serre. Les relations entre nos comportements quotidiens et les émissions de gaz à effet de serre sont évidentes dans quelques cas (par exemple, les émissions d'une voiture qui sont visibles au pot d'échappement), tandis que la plupart passent inaperçues en raison de la complexité du système d'élaboration et de consommation de produits et de services (par exemple, les émissions liées à la consommation de viande qui implique le déboisement de la forêt tropicale pour l'élevage et le transport, ou celles liées à la quantité d'intrants alimentaires, de l'eau et d'autres matériaux nécessaires à la production de chaque kilo de viande). L'invisibilité de la plupart des processus qui nous relient à ce problème contribue à favoriser l'inaction personnelle.

\section{L'incohérence entre les messages et les réponses politiques}

Le désir de passer à l'action dans la sphère personnelle se retrouve le plus souvent en porte-à-faux avec les politiques publiques et les messages optimistes associés à la culture de consommation projetée par la publicité, le marketing et d'autres processus 
qui formatent les modes de vie. Cette contradiction affaiblit également la perception sociale de la menace et décourage l'adoption de comportements alternatifs, difficiles à réaliser dans la pratique en l'absence de conditions visant à les favoriser (par exemple l'utilisation du transport en commun ou la consommation d'énergie provenant de sources alternatives). Les citoyens ont tendance à percevoir la lenteur du développement des politiques en réponse au changement climatique comme le signe qu'il s'agit d'une menace de seconde importance ou d'un problème ne nécessitant pas vraiment d'actions urgentes et même, qu'il existe une marge confortable de temps pour agir (Meira, 2008).

\section{Le culte du « progrès » et l'optimisme anthropologique}

La mémoire limitée du passé et la vision linéaire de l'histoire qui situe la civilisation moderne au zénith de l'évolution humaine, constituent la base d'un culte voué au progrès, soutenu par le mythe que l'avenir sera toujours et nécessairement meilleur que le présent. Ce mythe suppose la croyance que notre espèce a su surmonter par le passé d'autres moments critiques, alors que l'histoire humaine est remplie de civilisations effondrées et d'autres ayant connu des déclins et reculs notables (Diamond, 2005; Berman, 2007). Pour plusieurs d'entre elles, ce fut en raison de déséquilibres écologiques, et ce malgré des réalisations extrêmement sophistiquées à leur époque et dans leur contexte spécifique. Ce mythe se nourrit aussi de la confiance accordée en la science et la technologie comme instruments propres aux sociétés avancées permettant de trouver des solutions qui ne nécessitent pas de transformations substantielles du modèle établi.

\section{Les barrières psychosociales et de communication}

Le troisième type de déterminants de la représentation sociale du changement climatique a trait aux processus cognitifs et psychosociaux qui relaient l'information scientifique, la réinterprètent et l'intègrent dans la culture environnementale de la population. Il est nécessaire de les garder à l'esprit, au moins pour deux raisons :

a. afin de ne pas s'engager dans une candeur politique et pédagogique permettant de penser qu'il suffit de transférer l'information scientifique à propos du changement climatique vers la société -une alphabétisation scientifique conventionnelle - pour que ce problème soit compris et pris en charge, sans plus ;

b. pour aider à intégrer la prise en compte de la représentation sociale du changement climatique dans les programmes et les initiatives de communication, d'éducation et de participation visant à conscientiser et mobiliser la population au regard de la menace climatique.

Pour poursuivre, nous détaillerons brièvement certaines croyances, conceptions et certains préjugés plus répandus selon notre propre expérience de recherche (González Gaudiano, 2007 ; Gonzalez et Maldonado, 2013, Meira, 2008, Meira et autres, 2013) et celle systématisée par d'autres auteurs (Bord et al, 1998 ; Moser et Dilling, 2004 ; Weber, 2010) : 


\section{Un climat tempéré n'est-il pas préférable?} affectée par le changement climatique peut entretenir l'idée qu'il est possible d'espérer des bénéfices d'une augmentation de température qui peut sembler modérée ; après tout, il est question d'une augmentation de «seulement » quelques dixièmes de degré par décennie. De plus, les climats tempérés sont généralement perçus positivement dans la culture contemporaine des loisirs et du bien-être. Dans les latitudes plus froides et les communautés liées à des activités actuellement limitées par la variable thermique, la perspective d'une hausse de température pourrait être considérée comme un changement souhaitable (par exemple, pour la productivité et la qualité œnologique de certaines variétés de vigne ou pour la promotion du tourisme de soleil et de plage sur la côte atlantique européenne).

\section{Quand les sens nous « trompent »}

Notre appareil sensoriel est conçu pour capter l'évolution de la météo et pour répondre d'une manière adaptative aux variations thermiques quotidiennes et saisonnières. Mais il ne peut pas enregistrer les variations subtiles de la température, qui se mesurent en dixièmes de degré par décennie et s'échelonnent sur une échelle temporelle longue. Le réchauffement et la majeure partie des subtiles manifestations du changement climatique sont pour nous, en termes physiques, imperceptibles. L'acceptation que le changement est en marche devient littéralement une « question de foi » en la science. En fonction de notre culture, il est possible que des phénomènes météorologiques faisant partie de l'irrégularité propre à la météo habituelle, puissent être interprétés comme preuve ou réfutation du changement climatique, compte tenu de la nécessité que nous éprouvons à donner un sens à la réalité et à objectiver nos croyances à propos de celle-ci. Ainsi, une vague de froid sibérien dans une zone particulièrement sensible au changement climatique comme l'Europe du Sud peut être interprétée comme une preuve réfutant réchauffement du climat, alors qu'une vague de chaleur dans la même région peut laisser supposer la preuve du contraire, même si les deux peuvent constituer des événements météorologiques normaux pour ces latitudes.

\section{Barrières cognitives liées au traitement de l'information}

41 Si les sens montrent peu de sensibilité aux signes physiques du changement climatique, notre capacité à traiter l'information est également limitée et obéit à des principes d'économie et de simplification au sein de la vie quotidienne. Ces limites cognitives, s'ajoutant à l'incertitude, à la complexité et à l'ampleur du changement climatique, constituent une importante entrave à sa perception et sa représentation par la population.

42 Comme mentionné précédemment, passant à travers le filtre de la culture commune, l'information scientifique est traitée suivant des modèles épistémologiques différents de ceux qui régissent le domaine scientifique. La documentation spécialisée identifie certaines balises sociocognitives qui, depuis cette perspective, déforment la 
représentation du changement climatique et l'évaluation de sa menace potentielle par la population (Grothmann et Patt, 2005 ; Dietz et Stern, 2002 ; Jaspal et autres, 2014) :

a. La tendance à percevoir l'atmosphère comme un espace immense et vide, capable d'absorber tout et qui est resté inchangé et inchangeable au fil du temps. Cette croyance commune contraste avec le constat scientifique que l'atmosphère demeure un système fragile, constitué de plusieurs couches dont l'épaisseur totale est proportionnellement très faible par rapport au volume de la Terre et dont la composition et la dynamique se sont transformées tout au long de l'histoire naturelle de la planète.

b. La confusion entre la météo et le climat. Si nous pouvons quotidiennement expérimenter des changements de température de plusieurs degrés entre les minimums et maximums, et que nous y réagissons sans altérer substantiellement nos vies, comment une augmentation décimale de la température moyenne de la planète, sur une période de temps beaucoup plus longue, pourrait-elle être considérée comme une menace significative? La confusion fréquente entre la météo et le climat nuit à une représentation plus adéquate du changement climatique.

c. La tendance à penser que le réchauffement climatique est un processus linéaire et à miser sur le fait que les changements qui se produisent suivront une évolution graduelle dans le temps qui permettra d'adopter des stratégies de réponse et d'adaptation tout aussi progressives.

d. La tendance à insister sur l'importance des événements environnementaux extrêmes. Nous avons beaucoup de difficulté à saisir les changements graduels et progressifs (Weber, 2010 ; Bellamy et Hulme, 2011 ; Spence et al, 2012).

e. La croyance que l'action individuelle est négligeable devant l'ampleur du problème. Ce sentiment d'impuissance constitue l'une des principales barrières psychosociales qui empêchent le passage de la prise de conscience du changement climatique à l'action responsable. Même lorsque le problème est perçu, la réponse individuelle peut être considérée comme inadéquate et inutile compte tenu du défi épique que représente ce problème.

f. La construction de la représentation sociale du changement climatique à partir d'idées, de croyances et de conceptions relatives à la crise environnementale en général et à d'autres problèmes environnementaux plus spécifiques. La difficulté qu'éprouve une majorité de la population à comprendre le changement climatique ( $\mathrm{du}$ domaine scientifique) est compensée par l'activation de processus sociocognitifs qui permettent de recycler des idées et des représentations partagées à propos d'autres questions analogues.

43 L'exemple le plus saillant est la croyance courante selon laquelle il existe une relation de causalité entre la destruction de la couche d'ozone et le changement climatique (Reynolds et al, 2010 ; Ungar, 2000). Cette croyance est si répandue que l'on peut parler d'un "universel culturel» dans les sociétés dites avancées. Il s'agit d'un exemple paradigmatique de la façon dont les images, les informations et les concepts générés au sein du domaine scientifique s'intègrent et se réinterprètent dans la sphère de la culture commune. La façon dont les citoyens utilisent les connaissances socialement établies pour rendre intelligible le changement climatique, qu'elles soient valables scientifiquement ou non, peut constituer un obstacle important pour envisager les réponses politiques puisque, du point de vue de l'éducation et de la communication, il est plus simple de créer une nouvelle représentation que d'en modifier une déjà établie (Adams, 2001 ; Smith et Joffe, 2013). 


\section{Le coût perçu du changement}

44 appréciés ou désirés par la population, la perception du coût personnel associé à l'adoption de changements significatifs en termes de renoncement à un certain niveau de bien-être est très élevée. Soumis à la tension générée par la contradiction entre la conscience du problème et l'inaction personnelle, les gens transfèrent souvent vers d'autres acteurs la responsabilité de l'origine du problème et également, par conséquent, la recherche de solutions et d'alternatives: vers les industries, les gouvernements, les groupes de pression, les organisations internationales, les politiciens, etc. (Lenzen, 2001). Ainsi, la responsabilité individuelle est diluée dans la responsabilité collective, illustrant les paradoxes de la «tragédie des biens communs » (Hardin, 1968) : lorsque l'action personnelle implique un sacrifice d'une part de bienêtre individuel (objectif ou subjectif) au bénéfice des autres, l'inaction est justifiée et légitimée par l'inaction collective, soit l'inaction des autres, ou par l'inefficacité perçue des réponses institutionnelles.

\section{Le changement climatique et la hiérarchie des besoins}

Le changement climatique apparaît dans un contexte où plusieurs autres problèmes atteignent les personnes en tant que menaces globales ou locales : du terrorisme à la crise financière, en passant par les inégalités Nord-Sud, la menace d'effondrement de l'État-providence, le chômage ou l'insécurité publique. L'état permanent de crise fait en sorte que le sentiment d'insécurité fait désormais partie de la normalité et cela complique la prise de décision personnelle à propos de la priorité qu'il faut accorder à telle ou telle menace. Le changement climatique ne figure pas au premier rang parmi les préoccupations des gens. Ils sont plus attentionnés à d'autres problèmes plus aigus dans leur vie quotidienne. Cette échelle de priorités favorise la tendance à éviter et à repousser les problèmes qui sont perçus comme plus abstraits, indirects et lointains (Meira, 2008, Meira et autres, 2013).

\section{Éduquer en fonction des représentations sociales du changement climatique : un épilogue}

Il importe d'étudier plus avant la manière dont les représentations sociales des problèmes environnementaux globaux se construisent au sein des différents groupes et contextes sociaux, et pas seulement les groupes d'étudiants ou les milieux scolaires. Une meilleure compréhension de la façon dont la culture commune interprète, donne du sens, élabore des théories implicites et des croyances, et les utilise pour guider l'action peut contribuer à améliorer les processus éducatifs à propos de ces questions. Cela implique d'aller au-delà des approches transmissives et de la recherche constructiviste classique, centrées sur l'identification et la correction des erreurs qui se retrouvent dans les idées "scientifiques" des personnes. Les perspectives sociopédagogique et psychosociale doivent être prises en compte et renforcées. La recherche devrait également se diriger vers l'analyse comparative des représentations sociales du changement climatique dans les différentes sociétés, les groupes et les cultures. 
47 Nous devons aussi réviser le slogan "agir localement, penser globalement » comme guide pour l'action éducative et environnementale. Les problèmes environnementaux mondiaux nous obligent à penser et à agir simultanément dans les deux dimensions. L'examen de ce principe dans la pratique éducative présente un grand potentiel. Il s'agit de commencer par identifier dans chaque contexte social spécifique - au niveau régional et local - les liens entre les pratiques quotidiennes individuelles et collectives, et les causes et les conséquences du changement climatique. Il importe de cerner l'écart entre cette expérience sociale et la complexité de la nouvelle réalité mondiale. Par exemple, l'analyse de la généalogie sociale et environnementale des produits de consommation, souvent cachée, déformée ou ignorée par la puissante industrie culturelle du marketing, peut contribuer à cette tâche.

Il est nécessaire d'introduire le débat dans les contextes de la vie quotidienne, conçu comme une dynamique d'échange, une confrontation critique et une négociation de sens à propos des problèmes mondiaux. L'interaction sociale est au cœur du processus dynamique de construction des représentations sociales du changement climatique. Malheureusement, les problèmes environnementaux mondiaux constituent rarement des opportunités d'échange symbolique avec les autres, sauf occasionnellement, en contextes éducatifs formels ou parfois, en situations d'urgence.

Il importe de faire en sorte que les citoyens évitent de considérer a priori que les informations scientifiques ou autres qu'ils reçoivent sont valides, complètes et indiscutables. Par ailleurs, dans ce débat - dans les réunions de voisinage, les associations culturelles ou professionnelles, les regroupements de consommateurs, dans les espaces culturels, etc.-, nous ne devrions pas considérer seulement le problème du changement climatique, mais nous devrions examiner aussi les solutions possibles, tant à une échelle globale qu'à une échelle individuelle et ce, en articulant les deux. Il est nécessaire de promouvoir l'idée que le changement climatique n'est pas une question exclusivement scientifique et que les solutions devraient également provenir de la construction collective de nouvelles normes économiques, sociales, culturelles et éthiques. Il est donc souhaitable de créer des situations éducatives dans lesquelles le sujet-observateur ou détenteur d'information devient sujet-interprète et acteur social.

Compte tenu de ces défis, nous, éducateurs en environnement, devons également revoir nos représentations du changement climatique - informations, connaissances, interprétations, expériences, théories implicites, croyances, valeurs, dispositions pour l'action, etc. - afin de clarifier et de guider nos pratiques. Aussi, nous devrions être conscients que les destinataires de notre travail ont déjà leurs propres idées à propos $\mathrm{du}$ changement climatique et de la façon d'agir ou non face à lui. Les personnes ne se transforment pas soudainement en recevant de nouvelles informations ou des informations plus proches de la vérité scientifique. Dans la représentation du monde interviennent aussi d'autres variables et processus quotidiens - culturels, affectifs, expérientiels, situationnels, sociaux, et autres - qu'il importe de mettre en lumière : ils constituent également un matériel et un contenu éducatif de grande importance. 


\section{BIBLIOGRAPHIE}

Adams, S. (2001). Views of the uncertainties of climate change : a comparison of high school students and specialists. Canadian Journal of Environmental Education, 6, 58-76.

Barnes, T. J. (1996). Logics of dislocation. Models, metaphors and meanings of economic space. New York : The Guilford Press.

Bedolla, C.A. et Moreno, H. (2011). La ética del cambio climático. Revista Relaciones de Internacionales de la UNAM, 110, 121-137.

Bellamy, R. et Hulme, M. (2011). Beyond the Tipping Point : Understanding Perceptions of Abrupt Climate Change and Their Implications. Weather, Climate and Society, 3, 48-60.

Berman, M. (2007). Dark ages America. The final phase of empire. New York : W. W. Norton.

Bord, R. J., Fisher, A. et O'Connor, R.E. (1998). Public perceptions of global warming : United States and international perspectives. Climate Research, 11, 75-84.

Boykoff, M.T. (2011). Who Speaks for the Climate? Making Sense of media Reporting on Climate Change. Cambridge : Cambridge University Press.

Clark, F. J., Stamm, K. R. et Reynolds, P. (1998). Mass communication and public understaning of environmental risks : the case of global warming. Society for Risks Analisys 2008 Annual Meeting, 7-10 de diciembre, Boston, MA.

COP21 - Conference of the Parties (2015). Adoption of the Paris Agreement. ONU - Convention Cadre sur le Changement Climatique. http://unfccc.int/resource/docs/2015/cop21/eng/l09.pdf. Consulté le 12-12-2015.

Diamond, J. (2005). Collapse : how societies choose to fail or succeed, New York : Vicking-Penguin.

Dietz, T. et Stern, P.C. (2002). New tools for environmental protection, education, information, and voluntary measures, Washington DC : National Academy Press.

European Comission (2015). Special Eurobarometer 435. Climate Change. http://ec.europa.eu/ clima/citizens/support/docs/report_2015_en.pdf. Consulté le 8-12-2015.

Flannery, T. (2006). We are the weather makers. The story of global warming, Boston : Atlantic Monthly Press.

Foladori, G. (2000). El pensamiento ambientalista, Tópicos en Educación Ambiental, 2 (5), 21-38.

Gilmore, M. (2000). Ten illusions that must be dispelled before people will act on your global warming message, Climate Change Communication. Proceedings of an International Conference, 20-24 de junio, Kitchener-Waterloo, Ontario, Canada.

González Gaudiano, E. (2007). Educación y cambio climático : un desafío inexorable. Trayectorias. 25, 33-44.

González Gaudiano, E. et Maldonado González, A. L. (2013). Los jóvenes universitarios y el cambio climático. Xalapa : Universidad Veracruzana.

Grothmann, T. et Patt, A. (2005). Adaptive capacity and human cognition : the process of individual adaptation to climate change. Global Environmental Change, 15 (3), 199-213.

Hardin, G. (1968). The tragedy of commons. Science, 162 (3859), 1243-1248. 
Hempel, M. (2014). Ecoalfabetización : el conocimiento no es suficiente. En Prugh, T. y Renner, M. (Dirs.). Gobernar para la sostenibilidad. La situación del mundo 2014. Informe anual del Worldwach Institute sobre la sostenibilidad. Barcelona : Icaria.

Heras, F. et Meira. P. Á. (2014). ¿Cómo podemos mejorar la calidad de la información sobre el cambio climático ? En León, B. (Coord.). Periodismo, medios de comunicación y cambio climático. Salamanca : Comunicación Social, 28-58.

Hitchings, R. (2011). Coping with the immediate experience of climate : regional variations and indoor trajectories. WIREs Climate Change, 2 (2), 170-184.

IPCC (2014). Climate Change 2014. IPCC Fifth Assessment Synthesis Report. Summary for Policymakers. Geneva : IPCC.

Jaspal, R. ; Nerlich, B. et Cinnirella, M. (2014). Human Responses to Climate Change : Social Representation, Identity and Socio-psychological Action, Environmental Communication, 8 (1), 110-130.

Kahan, D.M., Peters, E., Wittlin, M., Slovic, P., Ouellette, L.L., Braman, D. et Mandel, G. (2012). The polarizing impact of science literacy and numeracy on perceived climate change risks. Nature Climate Change, 2, 732-735.

Leiserowitz, A. A. (2005). American Risk Perceptions : Is Climate Change Dangerous? Risk Analysis, 25 (6), 1443-1442.

Lenzen, M. (2001). The role of equity and lifestyles in education about climate change : experiences from a large-scale teacher development program. Canadian Journal of Environmental Education, 6, 32-51.

Lomborg, B. (2001). The skeptical environmentalist : measuring the real state of the world, Cambridge : Cambridge University Press.

Martínez Alier, J. (2007), “El ecologismo popular”, Ecosistemas. 16 (3), 148-151.

McBean, A. et Hengeveld, H.G. (2000). Communicating the science of climate change : a mutual challenge for scientists and educators, Canadian Journal of Environmental Education, 5 (1), 9-25.

Meira, P. (2008). Comunicar el cambio climático. Escenario social y líneas de actuación, Madrid : Ministerio de Medio Ambiente y Medio Rural y Marino.

Meira, P.Á. ; Arto, M. ; Heras, F. ; Iglesias, L. ; Lorenzo, J.J. et Montero, P. (2013). La respuesta de la sociedad española ante el cambio climático. 2013. Madrid : Fundación Mapfre.

Morgan, M., Fischhoff, B ; Bostrom, A. et Atman, C.J. (2002). Risk communication a mental model approach. Cambridge : Cambridge University Press.

Moscovici, S. et Hewstone, M. (1986). De la ciencia al sentido común. En Moscovici, S. (Ed.). Psicología social II. Barcelona : Paidós, 679-710.

Moser, S. C. et Dilling, L. (2004). Making climate hot : communicating the urgency and challenge of global climate change. Environment, 46 (10), 32-46.

Niemeyer, S., Petts, P. et Hobson, K. (2005). Rapid climate change and society. Assessing responses and thresholds, Risk Analysis, 25 (6), 1443-1456.

O'Connor, R. E., Bord, R.J. et Fisher, A. (1999). Risk perceptions, general environmental beliefs, and willingness to address climate change, Risk Analysis, 19 (3), 461-471.

Pape François (2015). Lettre Encyclique Laudato Si du Saint-Père François sur la sauvegarde de la maison commune. Vatican : Presses du Vatican 
Reynolds, T.W. ; Bostrom, A. ; Read, D. y Morgan, M.G. (2010). Now What Do People Know About Global Climate Change ? Survey Studies of Educated Laypeople. Risk Analysis, 30 (10), 1520-1538.

Scruggs, L. et Benegal, S. (2012). Declining public concern about climate change : Can we blame the great recession? Global Environmental Change, 22, 505-515.

Smith, N. et Joffe, H. (2013). How the public engages with global warming : A social representations approach. Public Understanding of Science, 22 (1), 16-32.

Spence,A. ; Poortinga, W. y Pidgeon, N. (2012) The Psychological Distance of Climate Change. Risk Analysis, 32 (6), 957-972.

Sterling, S. (2001). Sustainable education : re-visioning learning and change, Bristol : J. W. Arrowsmith.

Ungar, S. (2000). Knowledge, ignorance and the popular culture : climate change versus the ozone hole. Public Understanding of Science, 9, 297-312.

Urbina, J. et Martínez, J. (Comps.) (2006). Más allá del cambio climático. Las dimensiones psicosociales del cambio ambiental global, México : INE-UNAM.

Uzzell, D. L. (2000). The psycho-spatial dimension of global environmental problems, Journal of Environmental Psychology, 20 (4), 307-318.

Velayos, C. (208). Ética y cambio climático. Bilbao : Desclée De Brouwer.

Wagner, W. ; Hayes, N. y Flores, F. (2011). El discurso de lo cotidiano y el sentido común. La teoría de las representaciones sociales. Barcelona : Anthropos-UNAM.

Weber, E.U. (2010). What shapes perceptions of climate change ? WIREs Climate Change, 1, May/ June, 332-334.

Whitmarsh, L. (2011). Scepticism and uncertainty about climate change : Dimensions, determinants and change over time. Global Environmental Change, 21, 690-700.

Zehr, S.C. (2000). Public representations of scientific uncertainty about global climate change. Public Understanding of Science, 9, 85-103.

\section{RÉSUMÉS}

Les programmes éducatifs centrés sur la problématique du changement climatique sont le plus souvent axés sur des processus d'alphabétisation scientifique basés sur l'information issue des recherches en science du climat, sans considérer l'expérience sociale ni les dynamiques culturelles qui interviennent dans la construction des représentations sociales de ce phénomène. Or la prise en compte de cette dimension phénoménologique des réalités climatiques est essentielle pour relever adéquatement les défis de l'éducation et de la communication. Le principal obstacle au nécessaire changement social réside dans la nature structurelle de ce problème complexe, incluant ses dimensions morale, socio-politique, culturelle, socio-cognitive et psychosociale, qui conditionnent les représentations sociales et entravent l'adoption de changements significatifs dans les modes de vie individuels et collectifs liés aux activités qui bouleversent le climat. Dans cet article, trois types d'obstacles sont analysés et des propositions sont formulées afin de les surmonter.

Educational programs on this vital topic consist of processes of scientific literacy based on information about the findings of climate science, without considering the respective social experience or a series of social and cultural processes intervening in building a social 
representation of this phenomenon. That is essential for focusing properly on the challenges for education and communication. The main "barrier" to the necessary social change is the complex structural nature of the phenomenon, in which there are obstacles of a moral, socio-political, cultural, socio-cognitive and psycho-social nature. These are conditioning the social representations and hindering the adoption of significant changes in lifestyles, both individual and collective, related to human activities that imbalance climate. Three types of obstacles are analysed and proposals are posited to overcome them.

\section{INDEX}

Keywords : environmental education, climate change, cultural resistance, social obstacles, collective action

Mots-clés : éducation relative à l'environnement, changement climatique, résistances culturelles, obstacles sociaux, action collective

\section{AUTEURS}

\section{PABLO MEIRA}

Pablo Meira est professeur en éducation relative à l'environnement (Educación Ambiental) à l'Université de Santiago de Compostèle, en Espagne. Ses travaux portent principalement sur les fondements théoriques et les politiques publiques de l'éducation relative à l'environnement, sur les représentations sociales du changement climatique et sur l'éducation et la communication à la menace climatique. En 2009, il a reçu le Prix María Barbeito de Recherche en Pédagogie. Pablo Meira est actuellement directeur du Projet Resclima : www.resclima.info.

\section{ÉDGAR J. GONZÁLEZ GAUDIANO}

Édgar J. González Gaudiano est professeur et directeur de l'Institut de recherches en éducation de l'Université Veracruzana, au Mexique. Il coordonne la chaire UNESCO-UV en Citoyenneté, Éducation et Soutenabilité environnementale du Développement (Ciudadanía, Educación y Sustentabilidad Ambiental del Desarrollo). Ses travaux de recherche actuelle portent sur les représentations sociales du changement climatique, la vulnérabilité, le risque et la résilience sociale. 